\title{
Tratamento farmacológico de transtornos
} alimentares

Pharmacological Treatment of Eating Disorders

Fábio Tapia Salzano ${ }^{1}$

Tákı Athanássios CORdás ${ }^{2}$

\section{Resumo}

Os autores revisaram a literatura a respeito do tratamento farmacológico para transtornos alimentares, incluindo anorexia nervosa, bulimia nervosa e transtorno da compulsão alimentar periódica. São apresentadas evidências clínicas relacionadas ao uso de psicofármacos nos transtornos alimentares e apontadas, ainda, as perspectivas futuras para o tratamento.

Palavras-chave: Transtorno alimentar, tratamento; psicofarmacologia.

\section{Abstract}

The authors have revised the literature about the pharmacological treatment of eating disorders, including anorexia nervosa, bulimia nervosa and bingeeating disorder. Clinical evidences of the medications action in eating disorders are presented, and future perspectives for the treatment are indicated.

Keywords: Eating disorder, treatment, psychopharmacology. Alimentares do Ipq, Hospital das Clínicas da Faculdade de Medicina da Universidade de São Paulo - AMBULIM-Ipq HC-FMUSP, mestre pelo Departamento de Psiquiatria da FMUSP.

2 Coordenador geral do AMBULIM do Ipq HC-FMUSP, professor colaborador do Departamento de Psiquiatria da FMUSP.

Trabalho elaborado no AMBULIM do Ipq - HC-FMUSP, situado na rua Ovídio Pires de Campos s/n, $2^{\circ}$ andar - São Paulo - SP - CEP 05403-010, Fone/fax: 3069-6975.

Endereço para correspondência: Fábio Tapia Salzano. Ambulatório de Bulimia e Transtornos Alimentares do Ipq HC-FMUSP (AMBULIM), rua Ovídio Pires de Campos s/n, $2^{\circ}$ andar - São Paulo - SP- CEP 05403-010, Fone/fax: 3069-6975, e-mail: ftsalzano@uol.com.br. 


\section{Introdução}

A principal meta do tratamento da anorexia nervosa (AN), segundo a Associação Psiquiátrica Americana (APA, 2000), é o ganho de peso até o índice de massa corporal (IMC) acima de 19. Caso haja comorbidade psiquiátrica, é necessária a abordagem psicofarmacológica.

A meta principal do tratamento da bulimia nervosa (BN) é a regularização do padrão alimentar com suspensão de práticas purgativas e restritivas (APA, 2000). Para a maioria de pacientes com bulimia nervosa, um tratamento não-farmacológico é considerado o primeiro passo. Se o tratamento com abordagem psicoterápica não estiver evoluindo como o esperado, medicamentos antidepressivos estão indicados.

Os pacientes com transtorno da compulsão alimentar periódica (TCAP) são grupo de risco para ganho de peso e, à medida que o quadro evolui, atingem patamares de sobrepeso ou de obesidade. Em um estudo populacional, Fairburn et al. (2000) observaram que a taxa de pacientes com TCAP que eram obesos aumentou de $22 \%$ para $39 \%$ após cinco anos de evolução. Os objetivos do tratamento do TCAP devem incluir a redução dos episódios bulímicos, diminuição do peso corporal para pacientes obesos e melhora da psicopatologia associada (sintomas depressivos e ansiosos), preferencialmente com associação de psicofármacos e de psicoterapia (Stunkard e Allison, 2003). As três classes de medicamentos que estão sendo estudadas no TCAP são: antidepressivos, inibidores de apetite com ação no sistema nervoso central e anticonvulsivantes.

\section{Anorexia nervosa}

Nenhum psicofármaco foi claramente mais eficaz do que o placebo em melhorar os sintomas exclusivos daAN.

Um resumo de estudos controlados com placebo para AN pode ser observado na Tabela 1 .

Estudos com sulpirida (Vandereycken, 1984), carbonato de lítio (Gross et al., 1981), pimozida (Vandereycken \& Pieerloot, 1982), tetrahidrocanabinol (Gross et al., 1983), clonidina (Casper et al., 1987) e cisaprida (Stacher et al., 1993) não apresentaram resultados favoráveis.

O uso de amitriptilina (Biederman et al., 1985) e de ciproeptadina (Halmi et al., 1986) favoreceu o ganho de peso para pacientes com AN, enquanto a ação da fluoxetina mostrou resultados contraditórios (Gwirtsman et al., 1990; Attia et al, 1998).

Um estudo controlado comparou o uso de placebo e fluoxetina (doses entre 20 a $60 \mathrm{mg} / \mathrm{dia}$ ) em 35 pacientes com AN durante um ano (Kaye et al., 2001). Os resultados apontaram ganho de peso e melhora na psicopatologia da AN, no humor disfórico e nos pensamentos obsessivos somente para pacientes que utilizaram fluoxetina, sugerindo o uso da droga na prevenção de recaídas.

Tabela1. Estudos controlados para anorexia nervosa.

\begin{tabular}{|c|c|c|c|c|}
\hline Estudo & $\mathrm{N}$ & Duração - semanas & Medicamento & Resultado \\
\hline \multicolumn{5}{|l|}{ Ciproeptadina } \\
\hline $\begin{array}{l}\text { Vigersky \& Loriaux } \\
\text { (1977) }\end{array}$ & 24 & 8 & $\begin{array}{c}\text { Ciproeptadina } 12 \mathrm{mg} \\
\text { Placebo }\end{array}$ & Sem diferenças \\
\hline Goldberg et al. (1979) & 81 & Variada & $\begin{array}{c}\text { Ciproeptadina var. } \\
\text { Placebo }\end{array}$ & Sem diferenças \\
\hline Halmi et al. (1986) & 72 & 4 & Ciproeptadina $32 \mathrm{mg}$ & Ciproeptadina $>$ placebo \\
\hline \multicolumn{5}{|l|}{ Antidepressivos } \\
\hline Lacey \& Crisp (1980) & 16 & Variada & $\begin{array}{c}\text { Clomipramina } 50 \mathrm{mg} \\
\text { Placebo }\end{array}$ & Sem diferenças \\
\hline Biederman et al. (1985) & 25 & 5 & $\begin{array}{c}\text { Amitriptilina } 160 \mathrm{mg} \\
\text { Placebo }\end{array}$ & Sem diferenças \\
\hline Halmi et al. (1986) & 72 & 4 & $\begin{array}{c}\text { Amitriptilina } 175 \mathrm{mg} \\
\text { Placebo }\end{array}$ & Amitriptilina > placebo \\
\hline \multicolumn{5}{|l|}{ Antipsicóticos } \\
\hline $\begin{array}{l}\text { Vandereycken \& } \\
\text { Pierloot (1982) }\end{array}$ & 18 & 6 & $\begin{array}{c}\text { Pimozida } 6 \mathrm{mg} \\
\text { Placebo }\end{array}$ & Sem diferenças \\
\hline Vandereycken & 18 & 6 & $\begin{array}{c}\text { Sulpirida } 400 \mathrm{mg} \\
\text { Placebo }\end{array}$ & Sem diferenças \\
\hline \multicolumn{5}{|l|}{ Outros } \\
\hline Gross et al. (1981) & 16 & 4 & Carbonato de lítio & Sem diferenças \\
\hline Gross et al. (1983) & 11 & 4 & Tetrahidrocanabinol $30 \mathrm{mg}$ & Sem diferenças \\
\hline Casper et al. (1987) & 4 & 8 & Clonidina 0,5 a $0,7 \mathrm{mg}$ & Sem diferenças \\
\hline Stacher et al. (1983) & 12 & 12 & Cisaprida $30 \mathrm{mg}$ & Sem diferenças \\
\hline
\end{tabular}


Mendelson (2001) descreveu melhora do quadro clínico de uma paciente de 19 anos, com diagnóstico de anorexia nervosa subtipo restritivo, após o uso de tramadol, um agonista opióide seletivo.

Ouso da olanzapina ainda incipiente tem apresentado melhora na ansiedade, na recusa alimentar e no ganho de peso em estudos abertos (Malina et al., 2003).

\section{Bulimia nervosa}

O uso de antidepressivos, principalmente os tricíclicos e os inibidores seletivos de recaptura da serotonina (ISRS), tem auxiliado no tratamento da bulimia nervosa com a redução da freqüência de episódios bulímicos e vômitos, além de atuar em sintomas ansiosos e depressivos, quando presentes (APA, 2000). Os inibidores da monoaminoxidase (IMAO) também são úteis (Kennedy et al., 1988; Walsh et al., 1988; Mitchell et al., 1989-b; Priest et al., 1995), porém o risco de ingestão de algum alimento proscrito pelo seu uso durante um possível episódio faz a cautela anteceder sua indicação.

Em uma metanálise com 16 estudos controlados e um total de 1.300 pacientes com bulimia nervosa, Bacaltchuck et al. (2000) observaram maior remissão dos episódios bulímicos em pacientes medicados com antidepressivos do que com placebo (19,2\% contra 8\%) (Bacaltchuk et al., 2000). Em ambos os grupos, porém, as taxas de abandono foram consideradas altas (34,6\% para o grupo que usou antidepressivo e $31,4 \%$ para o grupo placebo), sem diferenças estatisticamente significativas entre eles.

Na Tabela 2, estão descritos os estudos controlados com placebo para bulimia nervosa.

Estudos com naltrexona (Mitchell et al., 1989); d-fenfluramina (Fahy et al., 1993) e carbonato de lítio (Hsu et al., 1991) não evidenciaram melhora na sintomatologia da bulimia nervosa.

O benefício com o uso da desipramina e imipramina no tratamento da bulimia nervosa foi observado por Hughes et al. (1986) e Pope et al. (1983), porém o uso de outro tricíclico, a amitriptilina, não apresentou resultados favoráveis.

Em 1992, um estudo multicêntrico (FBNC, 1992) de 387 pacientes com bulimia nervosa, por oito semanas e divididos em três grupos - um recebeu placebo, outro $20 \mathrm{mg} /$ dia de fluoxetina, e o terceiro, 60 $\mathrm{mg} /$ dia de fluoxetina - evidenciou uma redução significativa de sintomas bulímicos apenas para o terceiro grupo, com redução de $67 \%$ dos episódios bulímicos e vômitos auto-induzidos.

Em função desse estudo, Goldstein et al. (1995) realizaram outro experimento, comparando o uso de placebo com $60 \mathrm{mg} /$ dia de fluoxetina, demonstrando maior remissão de vômitos e episódios bulímicos, com significância estatística no grupo com a droga ativa.
Romano et al. (2002) observaram que tratamento de manutenção com $60 \mathrm{mg} /$ dia de fluoxetina por 52 semanas em pacientes com bulimia nervosa reduz o risco de recaída.

Em 2001, Knable descreveu remissão completa dos sintomas da bulimia nervosa em uma paciente com epilepsia, após o uso de topiramato, na dose de $150 \mathrm{mg} / \mathrm{dia}$.

Hoopes et al. (2003) avaliaram o uso de topiramato $(\mathrm{N}=35)$ em pacientes com bulimia nervosa num estudo duplo-cego controlado com placebo $(\mathrm{N}=34)$. A dose média de topiramato utilizada foi de $100 \mathrm{mg} / \mathrm{dia}$, variando de 25 a $400 \mathrm{mg} / \mathrm{dia}$. Os pacientes tratados com topiramato apresentaram redução de $44,8 \%$ no número médio de dias da semana com compulsão alimentar e/ou práticas purgativas, enquanto essa redução foi de 10,7\% para o grupo que utilizou placebo. Houve remissão completa ou grande redução dos episódios bulímicos e das práticas purgativas em nove dos 31 pacientes que utilizaram topiramato $(29 \%)$, contra dois dos 33 pacientes tratados com placebo (6,1\%). Esse estudo comprova a eficácia do topiramato na melhora da sintomatologia de pacientes com bulimia nervosa.

Em estudo aberto, El-Giamal et al. (2003) avaliaram 16 pacientes com bulimia nervosa, tratados com $100 \mathrm{mg} /$ dia de milnaciprano. Houve redução de pelo menos 50\% dos episódios bulímicos em dez dos 16 pacientes, e, ainda, três deles tiveram remissão completa ao final das oito semanas do estudo.

Igualmente em estudo aberto, Sloan et al. (2004) encontraram resultados positivos em 18 mulheres tratadas por oito semanas com doses de até $200 \mathrm{mg}$ de sertralina.

Não foram encontrados fatores preditivos de resposta consistentes no tratamento da bulimia nervosa até o momento (Hay e Bacaltchuk, 2001).

\section{Transtorno da compulsão alimentar periódica (TCAP)}

O uso de antidepressivos para pacientes com TCAP vem sendo avaliado a partir da observação de características clínicas semelhantes às dos pacientes com bulimia nervosa e da alta prevalência de comorbidade com episódios depressivos, que pode ocorrer em 51\% dos casos (Yanovski et al., 1993).

Os estudos controlados com placebo para o TCAP estão relacionados na Tabela 3.

Em 1990, McCann e Agras avaliaram o uso de desipramina por meio de um estudo controlado com placebo e observaram redução significativa na freqüência de episódios compulsivos, sem, entretanto, redução do peso corporal.

Alger et al. (1991) estudaram a ação de imipramina, naltrexona e placebo num estudo controlado para pacientes com TCAP, sem encontrar diferenças significativas na evolução dos três grupos. 
Tabela 2. Estudos controlados comparando medicamentos com placebo no tratamento da bulimia nervosa.

\begin{tabular}{|c|c|c|c|c|c|}
\hline Estudo & Medicação & $\begin{array}{c}\text { N Medicados } \\
\text { Completaram } \\
\text { (Randomizados) }\end{array}$ & $\begin{array}{c}\text { N Controles } \\
\text { Completaram } \\
\text { (Randomizados) }\end{array}$ & $\begin{array}{c}\% \\
\text { Mudança } \\
\text { E.B } \\
\text { Medicados }\end{array}$ & $\begin{array}{c}\% \\
\text { Mudança } \\
\text { E.B. } \\
\text { Controles }\end{array}$ \\
\hline \multicolumn{6}{|l|}{ Tricíclicos } \\
\hline Pope et al., 1983 & Imipramina & $9(11)$ & $10(11)$ & -70 & 0 \\
\hline Mitchell e Groet, 1984 & Amitriptilina & $16(21)$ & $16(17)$ & -72 & -52 \\
\hline Huges et al., 1986 & Desipramina & $7(10)$ & $9(12)$ & -91 & +19 \\
\hline Agras et al., 1987 & Imipramina & $10(10)$ & $10(12)$ & -72 & -43 \\
\hline Barlow et al., 1988 & Desipramina & $24(47)$ & - & -47 & -2 \\
\hline Blouin et al., 1988 & Desipramina & $10(17)$ & - & -40 & - \\
\hline Mitchell et al., 1990 & Imipramina & $31(54)$ & $26(31)$ & -51 & 0 \\
\hline McCann e Agras, 1990 & Desipramina & $10(15)$ & $13(15)$ & -63 & -16 \\
\hline Alger et al., 1991 & Imipramina & (7) & (7) & -26 & -30 \\
\hline Walsh, 1991 & Desipramina & $31(40)$ & $32(38)$ & -47 & +7 \\
\hline Rotschild et al. 1994 & $\begin{array}{l}\text { Imipramina } \\
\text { Fenelzina }\end{array}$ & $\begin{array}{l}5(8) \\
3(6)\end{array}$ & $6(10)$ & - & - \\
\hline \multicolumn{6}{|l|}{ ISRS } \\
\hline Fichter et al., 1991 & Fluoxetina & 19(20) & $20(20)$ & - & - \\
\hline \multirow[t]{2}{*}{ FBNC, 1992} & Fluoxetina 20 & $98(129)$ & $79(129)$ & -45 & -33 \\
\hline & Fluoxetina 60 & $89(129)$ & & -67 & - \\
\hline Kanerva et al., 1995 & Fluoxetina & $22(24)$ & $24(26)$ & - & - \\
\hline Goldstein et al., 1995 & Fluoxetina & $170(296)$ & $49(102)$ & -50 & -18 \\
\hline \multicolumn{6}{|l|}{ IMAO } \\
\hline Walsh et al.,1988 & Fenelzina & $23(31)$ & $27(31)$ & -64 & -5 \\
\hline Kennedy et al., 1988 & Isocarboxazida & $18(29)$ & - & -35 & +5 \\
\hline \multicolumn{6}{|l|}{ Outros } \\
\hline Sabine 1983 & Mianserina & $14(20)$ & $22(30)$ & - & - \\
\hline Horne et al., 1988 & Bupropiona & $37(55)$ & $12(26)$ & -66 & -23 \\
\hline Pope et al., 1989 & Trazodona & $20(23)$ & $22(23)$ & -31 & +21 \\
\hline Kennedy et al., 1993 & Brofaromina & $15(19)$ & $13(17)$ & -62 & -50 \\
\hline Hoopes et al., 2003 & Topiramato & $19(31)$ & $17(33)$ & -49 & -28 \\
\hline
\end{tabular}

Ainda a respeito da naltrexona, há um relato de caso de uma adolescente com TCAP apontando remissão dos episódios compulsivos quando o opióide foi associado à fluoxetina (Neumeister et al., 1999).

Um estudo aberto com fluvoxamina apontou melhora de episódios compulsivos e redução de peso em um grupo de pacientes obesos com TCAP (Gardiner et al., 1993).

Os estudos controlados com ISRS indicaram, até o momento, benefícios com uso de fluvoxamina (Hudson et al.,1998), sertralina (McElroy et al., 2000), fluoxetina (Arnold et al., 2002) e citalopram (McElroy et al., 2003) no TCAP, com redução nos episódios bulímicos.

Um estudo aberto com venlafaxina sugeriu a eficácia deste antidepressivo na melhora de episódios compulsivos (Malhotra et al., 2002).

Em razão da associação do TCAP com obesidade, o uso de um inibidor de apetite com ação no sistema nervoso central, em regiões que regulam a saciedade e o apetite, pode promover melhora no quadro clínico relacionado.

Stunkard et al. (1996) observaram melhora significativa nos episódios bulímicos, sem diminuição do peso em pacientes com TCAP com d-fenfluramina.

Após um estudo aberto com sibutramina (Appolinario et al., 2002) evidenciando resultados promissores, Appolinário et al. (2003) realizaram um estudo controlado com sibutramina (dose de $15 \mathrm{mg} /$ dia) e placebo em pacientes ambulatoriais com TCAP e obesidade. Ao final de 12 semanas do estudo, houve redução de $63 \%$ dos episódios bulímicos para o grupo que usou a sibutramina e aumento de $5,7 \%$ deles para o grupo placebo. A melhora na pontuação das escalas Binge Eating Scale e Beck Depression Inventory foi maior para o grupo do medicamento. Houve redução média de $7,4 \mathrm{~kg}$ de peso no grupo da sibutramina e ganho de $1,4 \mathrm{~kg}$ no grupo placebo. 
Tabela 3. Estudos controlados comparando medicamentos com placebo no tratamento da compulsão alimentar periódica (TCAP).

\begin{tabular}{|c|c|c|c|c|c|c|c|}
\hline Estudo & $\begin{array}{l}\text { Medicamento, } \\
\text { Dose (mg) }\end{array}$ & Diagnóstico & $N$ & $\begin{array}{l}\text { Duração } \\
\text { (Semanas) }\end{array}$ & $\begin{array}{c}\text { Freqüência } \\
\text { EP }\end{array}$ & IMC & $\begin{array}{c}\text { Melhora } \\
\text { global }\end{array}$ \\
\hline $\begin{array}{l}\text { Tricíclicos } \\
\text { McCann \& Agras (1990) } \\
\text { Alger et al. (1991) }\end{array}$ & $\begin{array}{l}\text { Desipramina } 100-300 \\
\text { Imipramina } 150-200\end{array}$ & $\begin{array}{c}\text { BN (np) } \\
\text { TC, obesidade }\end{array}$ & $\begin{array}{l}23 \\
23\end{array}$ & $\begin{array}{c}12 \\
8\end{array}$ & $\begin{array}{l}\text { ES } \\
\text { NS }\end{array}$ & $\begin{array}{l}\text { NS } \\
\text { NS }\end{array}$ & $\begin{array}{l}\text { NA } \\
\text { NA }\end{array}$ \\
\hline $\begin{array}{l}\text { ISRS } \\
\text { McElroy et al. (in press) } \\
\text { Arnold et al. (2002) } \\
\text { Grilo (2002) } \\
\text { Hudson et al. (1998) } \\
\text { McElroy et al. (2000) }\end{array}$ & $\begin{array}{c}\text { Citalopram } 20-60 \\
\text { Fluoxetina } 20-80 \\
\text { Fluoxetina } 60 \\
\text { Fluvoxamina } 50-300 \\
\text { Sertralina } 50-200\end{array}$ & $\begin{array}{l}\text { TCAP } \\
\text { TCAP } \\
\text { TCAP } \\
\text { TCAP } \\
\text { TCAP }\end{array}$ & $\begin{array}{l}38 \\
60 \\
52 \\
85 \\
34\end{array}$ & $\begin{array}{c}6 \\
6 \\
16 \\
9 \\
6\end{array}$ & $\begin{array}{l}\text { ES } \\
\text { ES } \\
\text { NS } \\
\text { ES } \\
\text { ES }\end{array}$ & $\begin{array}{l}\text { ES } \\
\text { ES } \\
\text { NS } \\
\text { ES }\end{array}$ & $\begin{array}{l}\text { ES } \\
\text { ES } \\
\text { NA } \\
\text { ES } \\
\text { ES }\end{array}$ \\
\hline $\begin{array}{l}\text { Inibidores de apetite } \\
\text { Stunkard et al. (1996) } \\
\text { Appolinario et al. (2003) }\end{array}$ & $\begin{array}{c}\text { d-fenfluramina } 15-30 \\
\text { Sibutramina } 15\end{array}$ & $\begin{array}{c}\text { TCAP } \\
\text { TCAP, obesidade }\end{array}$ & $\begin{array}{l}28 \\
60\end{array}$ & $\begin{array}{c}8 \\
12\end{array}$ & $\begin{array}{l}\text { ES } \\
\text { ES }\end{array}$ & $\begin{array}{l}\text { NS } \\
\text { ES }\end{array}$ & $\begin{array}{l}\text { NA } \\
\text { NA }\end{array}$ \\
\hline $\begin{array}{l}\text { Anticonvulsantes } \\
\text { McElroy et al. (2003) }\end{array}$ & Topiramato $50-600$ & TCAP, obesidade & 58 & 14 & ES & ES & ES \\
\hline
\end{tabular}

ISRS: inibidor seletivo de recaptura de serotonina; Melhora global: avaliada pela Clinical Global Impression-Severity Scale; TC: transtorno compulsivo;

Adaptado de Carter et al. (2003) BN (np): bulimia nervosa não-purgativa; EP: episódios bulímicos; IMC: índice de massa corporal; ES: melhora estatisticamente significante com uso do medicamento frente ao placebo; NS: sem diferenças estatisticamente significantes entre o medicamento e o placebo; NA: não-avaliado

Alguns anticonvulsivantes como topiramato e zonisamida promovem diminuição de apetite e perda de peso em alguns pacientes, sendo estudados no tratamento do TCAP.

Em dois estudos abertos sobre topiramato no TCAP, houve redução dos episódios bulímicos e diminuição do peso corporal (Shapira et al., 2000; Appolinário et al., 2002).

Um estudo controlado comparando a ação do topiramato com placebo em 61 pacientes com TCAP e obesidade comprovou os achados descritos anteriormente (McElroy et al., 2003). Houve remissão completa dos episódios bulímicos em $64 \%$ do pacientes que utilizaram o topiramato (dose média de $212 \mathrm{mg} /$ dia) e em 30\% dos que usaram placebo.

A eficácia da zonisamida no TCAP foi comprovada em um único estudo aberto utilizando dose média de $513 \mathrm{mg} /$ dia, com redução no peso e do número de episódios compulsivos (McElroy et al., 2004).

\section{Conclusões}

A anorexia nervosa ainda não possui um tratamento farmacológico considerado eficaz para melhora da psicopatologia do transtorno.

A ação de antidepressivos, entretanto, na prevenção de recaídas e de antipsicóticos no ganho de peso pode sinalizar como deverão ser pesquisas futuras.

O uso de antidepressivos, principalmente tricíclicos e ISRS, mostra-se parcialmente eficaz na bulimia nervosa e no TCAP. Mais recentemente, a ação do topiramato vem adicionando renovadas esperanças para o tratamento de ambos os quadros e do TCAP.

Novas pesquisas são necessárias para determinação das doses eficazes e duração do tratamento, bem como diferenças nos resultados terapêuticos com associação de psicoterapia.

\section{Referências bibliográficas}

Agras, W.S.; Dorian, B.; KiRKley, B.G.; Arnow, B. \& Bachman, I.Imipramine in the treatment of bulimia: a double-blind controlled study. Int J Eat Disorder 6: 29-38, 1987.

Alger, S.A.; Schwalberg, M.D.; Bigaduette, J.M.; Michalek, A.V. \& HowARD, L.J.- Effect of a trcyclic antidepressant and opiate antagonist on binge-eating behavior in normoweight bulimic and obese binge-eating subjects. Am J Clin Nutrition 53: 865-71, 1991.
American Psychiatric Association (APA).- Practice guideline for the treatment of patients with eating disorders (revision). Am J Psychiatry 157 (suppl. 1): 1-39, 2000.

Appolinario, J.C.; Bacaltchuk, J.; Sichieri, R.; Claudino, A.; GodoyMatos, A.; Morgan, C.M.A. et al.- A randomized, doubleblind, placebo-controlled study of sibutramine in the treatment of binge-eating disorder. Arch Gen Psychiatry 60: 1109-16, 2003. 
Appolnario, J.C; Fontenelle, L.F; Papelbaum, M.; Bueno, J.R. \& Coutinho, W.- Topiramate use in obese patients with binge-eating disorder: an open study. Can J Psychiatry 47: 271-3, 2002.

Appolinario, J.C.; Godoy-Matos, A.; Fontenelle, L.F.; Carraro, L.; Cabral, M.; Vielra, A. \& Coutinho, W.- An open-label trial of sibutramine in obese patients with binge-eating disorder. J Clin Psychiatry 63: 28-30, 2002.

Arnold, L.M.; McElroy, S.L.; Hudson, J.I.; Welge, J.A.; Bennett, A.J. \& KECK, P.E., Jr.- A placebo-controlled randomized trial of fluoxetine in the treatment of binge-eating disorder. J Clin Psychiatry 63 1028-33, 2002.

AttiA, E.; Haiman, C.; Walsh, B.T. \& Flater, S.R.- Does fluoxetine augment the inpatient treatment of anorexia nervosa? Am J Psychiatry 155: 548-51, 1998.

BACALTCHUK, J.; HAY, P.J. \& MaRl, J.J.- Antidepressants versus placebo for the treatment of bulimia nervosa: a systematic review. Austr N Zeal J Psychiatry 34: 310-7, 2000.

Barlow, J.; Blouin, J.H.; BlouIn, A.G.; Perez., E.L.- Treatment of bulimia with desipramine: a double-blind crosspver study. Can J Psychiatry 33: 129-33, 1988.

Biederman, J.; Herzog, D.B.; Rivinus, T.M.; Harper, G.P.; Ferber, R.A.; Rosenbaum, J. et al.- Amitriptyline in the treatment of anorexia nervosa: a double-blind, placebocontrolled study. J Clin Psychopharmacol 5 :10-6, 1985.

Blouin, A.G.; Blouin, G.K.; Perez, E.L.; Bushnik, T.; Zuro, C. \& MULDER, E.- Treatment of bulimia with fenfluramine and desipramine. J Clin Psychopharmacol 8: 261-9, 1988.

CAsper, R.C.; Schlemmer, R.F. JR. \& Javaid, J.I.- A placebocontrolled crossover study of oral clonidine in acute anorexia nervosa. Psychiatry Res 20: 249-60, 1987.

El-Giamal, N.; de Zwaan, M.; Baller, U.; Strnad, A.; Schüssler, P. \& KASPER, S.- Milnacipran in the treatment of bulimia nervosa: a report of 16 cases. Eur Neuropsychopharmacol 13: 73-9, 2003.

FAhY, T.A.; EIsLer, I. \& RusselL, F.M.- A placebo-controlled trial of d-fenfluramine in bulimia nervosa. Br J Psychiatry 162: 597-603, 1993.

Faiburn, C.G.; Cooper, Z.; Doll, H.A.; Norman, P. \& O'Connor, M.The natural course of bulimia nervosa and binge eating disorder in young women. Arch Gen Psychiatry 57: 65965, 2000.

FBNC Study Group.- Fluoxetine in the treatment of bulimia nervosa: a multicenter, placebo-controlled, doubleblind trial. Arch Gen Psychiatry 49: 139-47, 1992.

Fichter, M.M.; LeibL, K.; Rief, W.; Brunner, E.; Schimidt-Aubeerger, S. \& Engel, R.R.- Fluoxetine versus placebo. A doubleblind study with bulimic inpatients under intensive psychotherapy. Pharmacopsychiatry 24: 1-7, 1991.

Gardiner, H.M.; Freeman, C.P.L.; Jesinger, D.K. \& Coluins, S.A.Fluvoxamine: an open pilot study in moderately obese female patients suffering from atypical eating disorders and episodes of bingeing. Int $J$ Obes Relat Metab Disord 17: 301-5, 1993.

Goldberg, S.C.; Halmi, K. \& Eckert, E.D.- Cyproheptadine in anorexia nervosa. Br J Psychiatry 134: 67-70, 1979.

Goldstein, D.J.;Wilson, M.G.; Thompson, V.L.; PotVIn, J.H.; Rampey, A.H., Jr. \& The Fluoxetine Bulimia Nervosa Research
Group.- Long-term fluoxetine treatment of bulimia nervosa. Br J Psychiatry 166: 660-6, 1995.

Gross, H.A.; Ebert, M.H.; Faden, V.B.; Goldberg, S.C.; Kaye, W.H.; CAINE, E.D. et al.- A double-blind trial of D9tetrahydrocannabinol in primary anorexia nervosa. $J$ Clin Psychopharmacol 3: 165-71, 1983.

Gross, H.A.; Ebert, M.H.; Faden, V.B.; Goldberg, S.C.; Nee, L.E. \& KAYE, W.H.- A double-blind controlled trial of lithium carbonate in primary anorexia nervosa. J Clin Psychopharmacol 1: 376-81, 1981.

GuirTSMAn, H.E.; GuzE, B.H.; YAGER, J. \& GaInsLeY, B.- Fluoxetine treatment of anorexia nervosa: an open clinical trial. $J$ Clin Psychiatry 51: 378-82, 1990.

Halmi, K.A.; ECKert, E.; LADu, T.J. \& Cohen, J.- Anorexia nervosa. Treatment efficacy of cyproheptadine and amitriptyline. Arch Gen Psychiatry 43: 177-81, 1986.

HaY, P.J. \& BaCALTCHUK, J.- Bulimia nervosa. Extracts from"Clinical Evidence". BMJ 323: 33-7, 2001.

Hughes, P.L.;Wells, L.A.; Cunningham, C.J. \& ILstrup, D.M.- Treating bulimia with desipramine. A double-blind, placebocontrolled study. Arch Gen Psychiatry 43: 82-6, 1986.

Hoopes, S.P.; ReimherR, F.W.; Hedges, D.W.; Rosenthal, N.R.; Kamin, M.; KARIM, R. et al.- Treatment of bulimia nervosa with topiramate in a randomized, double-blind, placebocontrolled trial, part 1: Improvement in binge and purge measures. J Clin Psychiatry 64: 1335-41, 2003.

Hsu, L.K.G.; Clement, L.; SAnthouse, R. \& Ju, E.S.Y.- Treatment of bulimia nervosa with lithium carbonate: a controlledstudy. J Nerv Ment Dis 179: 351-5, 1991.

Horne, R.L.; Ferguson, J.M.; PoPe, H.G., JR.; Hudson, J.I.; LineberRY, C.G.; AscheR, J. \& Cato, A.- Treatment of bulimia with bupropion: a multicenter controlled trial. J Clin Psychiatry 49: 262-6, 1988.

Hudson, J.I.; McElroy, S.L.; Raymond, N.C.; Crow, S.; Keck, P.E., JR.; CARTER, W.P. et al.- Fluvoxamine in the treatment of bingeeating disorder: a multicenter placebo-controlled, double-blind trial. Am J Psychiatry 155: 1756-62, 1998.

Hughes, P.L.; Wells, L.A.; Cunningham, C.J. \& ILSTRuP, D.M.- Treating bulimia nervosa with desipramine: a double-blind, placebocontrolled study. Arch Gen Psychiatry 43: 182-6, 1986.

Kanerva, R.; Rissanen, A. \& Sarna, S.- Fluoxetine in the treatment of anxiety, depressive symptoms, and eatingrelated symptoms in bulimia nervosa. Nordic $J$ Psychiatry 49: 237-42, 1995.

KAYE, W.H.- A double-blind controlled trial of lithium carbonate in primary anorexia nervosa. J Clin Psychopharmacol 1: 376-81, 1981.

Kaye, W.H.; Nagata, T.; Weltzin, T.E.; Hsu, L.K.G.; Sokol, M.S.; McConaha, C. et al.- Double-blind placebo-controlled administration of fluoxetine in restricting and restricting-purging-type anorexia nervosa. Biol Psychiatry 49: 644-52, 2001.

Kennedy, S.H.; Goldbloom, D.S.; Ralevski, E.; Davis, C.; D'Souza, J.D. \& LoFCHY, J.- Is there a role for selective monoamine oxidase inhibitor therapy in bulimia nervosa? A placebocontrolled trial of brofaramine. J Clin Psychopharmacol 13: 415-22, 1993. 
KnABLE, M.B.- Topiramate for bulimia nervosa in epilepsy. Am J Psychiatry 158: 322-3, 2001.

LACEY, J.H. E CRISP, A.H.- Hunger, food intake and weight: the impact of clomipramine on a refeeding anorexia nervosa population. Postgraduate Med J 56 (supplement 1): S79-S85, 1980.

Malhotra, S.; King, K.H.; Welge, J.A.; Brusman-Lovins, L. \& McELRoY, S.L.- Venlafaxine treatment of binge-eating disorder associated with obesity: a series of 35 patients. $J$ Clin Psychiatry 63: 802-6, 2002.

Malina, A.; Gaskill, J.; McConaha, C.; Frank, G.K.; LaVia, M.; Scholar, L. \& KAYE, W.H.- Olanzapine treatment of anorexia nervosa: a retrospective study. Int J Eat Disorder 33: 234-7, 2003.

McCann, U.D. \& Agras, W.- Successful treatment of nonpurging bulimia nervosa with desipramine: A doubleblind, placebo-controlled study. Am J Psychiatry 147: 1509-13, 1990.

McElroy, S.L.; Arnold, L.M.; Shapira, N.A.; KecK., P.E.; JR.; Rosenthal, N.R.; Karim, M.R.et al.- Topiramate in the treatment of binge-eating disorder associated with obesity: a randomized, placebo-controlled trial. $A m \mathrm{~J}$ Psychiatry 160: 255-61, 2003.

McElroy, S.L.; Arnold, L.M.; CAsuto, L.S.; Nelson, E.B.; Lake, K.A.; SoutuLto, C.A. et al.- Placebo-controlled trial of sertraline in the treatment of binge-eating disorder. $A m \mathrm{~J}$ Psychiatry 157: 1004-6, 2000.

McElroy, S.L.; Hudson, J.I.; Malhotra, S; Welge, J.A.; Nelson, E.B. \& KECK, P.E., Jr.- Citalopram in the treatment of bingeeating disorder: a placebo-controlled trial. J Clin Psychiatry 64: 807-13, 2003.

McElroY, S.L.; Kotwal, R.; Hudson, J.I.; Nelson, E.B. \& KecK, P.E., $\mathrm{Jr}$-- Zonisamide in the treatment of binge eating disorder: an open-label, prospective trial. J Clin Psychiatry 65: 50-6, 2004

Mendelson, S.D.- Treatment of anorexia nervosa with tramadol. Am J Psychiatry 158: 963-4, 2001.

Mitchell, J.E.; Christenson, G.; Jennings, J.; Huber, N.; Thomas, B.; Pomeroy, C. \& Morley, J.- A placebo-controlled, doubleblind crossover study of naltrexone hydrochlorie with normal weight bulimia. J Clin Psychopharmacol 9: 947,1989-a.

Neumeister, A.; Winkler, A. \& Wöber-Bingol, C.- Addition of naltrexone to fluoxetine in the treatment of binge eating disorder. Am J Psychiatry 156: 5, 1999.

Pope, H.G.; Hudson, J.I.; JonAS, J.M. \& YuRGelun-Todd, D.- Bulimia treated with imipramine: a placebo-controlled, doubleblind study. Am J Psychiatry 140: 554-8, 1983.
Pope, H.G.; Hudson, J.I.; Jonas, J.M. \& Yurgelun-Todd, D.Antidepressant treatment of bulimia: a two-year followup study. J Clin Psychopharmacol 5: 320-7, 1985.

Pope, H.G.; Keck, P.E., JR; McElroy, S.L. \& Hudson, J.I.- A placebo-controlled study of trazodone in bulimia nervosa. J Clin Psychopharmacol 9: 254-9, 1989.

Priest, R.G.; Gimbrett, R.; Roberts, R. \& Steinert, J.- Reversible and selective inhibitors of monoamine oxidase $A$ in mental and other disorders. Acta Psychiatr Scand 91 (suppl. 386): 40-3, 1995.

Romano, S.J.; Halmi, K.A.; SARKar, N,P.; Koke, S.C. \& Lee, J.L.- A placebo-controlled study of fluoxetine in continued treatment of bulimia nervosa after successful acute fluoxetina treatment. Am J Psychiatry 159: 96-102, 2002.

ROTSCHILD, R.; QUITKIN, H.M.; QUITKIN, F.M.; SteWART, J.W.; OCEPEKWelikson, K.; McGrath, P.J. \& Tricamo, E.- A double-blind placebo-controlled comparison of phenelzine and imipramine in the treatment of bulimia in atypical depressives. Int J Eat Disorder 15: 1-9, 1994.

Sabine, E.J.; Yonace, A.; Farrington, A.J.; Barrant, K.H. \& Wakeling, A.- Bulimia nervosa: a placebo-controlled double-blind therapeutic trial of mianserin. Br J Clin Pharmacol 15: 195-202, 1983.

ShapiRA, N.A.; Goldsmith, T.D. \& McElrov, S.L.- Treatment of binge eating disorder with topiramate: a clinical case series. J Clin Psychiatry 61: 368-72, 2000.

SLoAN, D.M.; Mizes,J.S.; HelBoK, C. \& Muck, R.-Efficacy of sertraline for bulimia nervosa, Int J Eat Disorder 36: 48-54, 2004.

Stacher,G.; Abatzl-Wenzel, T.A.; Wiesnagrotzki,S.; Bergmann, H.; SChneider, C. \& Gaupmann, G.- Gastric emptying, body weight and symptoms in primary anorexia nervosa. Long-term effects of cisapride. Br J Psychiatry 162: 398-402, 1993.

StUnKARD, A.J. \& ALISON, K.C.- Binge eating disorder: disorder or marker? Int J Eat Disorder 34: S107-S116, 2003.

Stunkard, A.J.; Berkowitz, R.; Tankikut, C.; Reiss, E. \& Young, L.$D$-fenfluramine treatment of binge eating disorder. $A m$ J Psychiatry 153: 1455-9, 1996.

VANDEREYCKEN, W.- Neuroleptics in the short-term treatment of anorexia nervosa: a double-blind placebo-controlled study with sulpiride. Br J Psychiatry 144: 288-92, 1984.

Vandereycken, W. \& Pierloot, R.- Pimozide combined with behavior therapy in the short-term treatment of anorexia nervosa. Acta Psychiatr Scand 66: 445-50, 1982.

Yanovski, S.Z;; Nelson, J.E.; DubBer, B.K. \& SPITER, R.L.- Association of binge eating disorder and psychiatric comorbidity in obese subjects. Am J Psychiatry 150: 1472-9, 1993. 